DRAFT VERSION NOVEMBER 7, 2018

Preprint typeset using LTEX style emulateapj v. 03/07/07

\title{
AN ENTROPY THRESHOLD FOR STRONG H $\alpha$ AND RADIO EMISSION IN THE CORES OF GALAXY CLUSTERS
}

\author{
Kenneth. W. Cavagnolo, Megan Donahue, G. Mark Voit, and Ming Sun \\ Michigan State University, Department of Physics and Astronomy, East Lansing, MI 48824; cavagnolo@pa.msu.edu \\ Draft version November 7, 2018
}

\begin{abstract}
Our Chandra X-ray Observatory archival study of intracluster entropy in a sample of 222 galaxy clusters shows that $\mathrm{H} \alpha$ and radio emission from the brightest cluster galaxy are much more pronounced when the cluster's core gas entropy is $\lesssim 30 \mathrm{keV} \mathrm{cm}^{2}$. The prevalence of $\mathrm{H} \alpha$ emission below this threshold indicates that it marks a dichotomy between clusters that can harbor multiphase gas and star formation in their cores and those that cannot. The fact that strong central radio emission also appears below this boundary suggests that AGN feedback turns on when the intracluster medium starts to condense, strengthening the case for AGN feedback as the mechanism that limits star formation in the Universe's most luminous galaxies.

Subject headings: conduction - cooling flows - galaxies: active - galaxies: clusters: general - X-rays: galaxies: clusters - galaxies: evolution
\end{abstract}

\section{INTRODUCTION}

In recent years the "cooling flow problem" has been the focus of intense scrutiny as the solutions have broad impact on our theories of galaxy formation (see Peterson \& Fabian 2006 for a review). Current models predict that the most massive galaxies in the Universe - brightest cluster galaxies (BCGs) - should be bluer and more massive than observations find, unless AGN feedback intervenes to stop latetime star formation (Bower et al. 2006; Croton et al. 2006; Saro et al. 2006). X-ray observations of galaxy clusters have given this hypothesis considerable traction. From the properties of X-ray cavities in the intracluster medium (ICM), Bîrzan et al. (2004) concluded that AGN feedback provides the necessary energy to retard cooling in the cores of clusters (see McNamara \& Nulsen 2007 for a review). This result suggests that, under the right conditions, AGN are capable of quenching star formation by heating the surrounding ICM.

If AGN feedback is indeed responsible for regulating star formation in cluster cores, then the radio and star-forming properties of galaxy clusters should be related to the distribution of ICM specific entropy ${ }^{1}$. In previous observational work (see Donahue et al. 2005, 2006; Cavagnolo et al. 2008a), we have focused on ICM entropy as a means for understanding the cooling and heating processes in clusters because it is a more fundamental property of the ICM than temperature or density alone (Voit et al. 2002; Voit 2005). ICM temperature mainly reflects the depth and shape of the dark matter potential well, while entropy depends more directly on the history of heating and cooling within the cluster and determines the density distribution of gas within that potential.

We have therefore undertaken a large Chandra archival project to study how the entropy structure of clusters correlates with other cluster properties. Cavagnolo et al. (2008a) presents the radial entropy profiles we have measured for a sample of 222 clusters taken from the Chandra Data Archive. We have named this project the Archive of Chandra Cluster Entropy Profile Tables, or ACCEPT for short. To characterize the ICM entropy distributions of the clusters, we fit the equation $K(r)=K_{0}+K_{100}(r / 100 \mathrm{kpc})^{\alpha}$ to each entropy profile. In

\footnotetext{
${ }^{1}$ In this paper we quantify entropy in terms of the adiabatic constant $K=$ $k T n_{e}^{-2 / 3}$.
}

this equation, $K_{100}$ is the normalization of the power-law component at $100 \mathrm{kpc}$ and we refer to $K_{0}$ as the central entropy. Bear in mind, however, that $K_{0}$ is not necessarily the minimum core entropy or the entropy at $r=0$, nor is it the gas entropy which would be measured immediately around the AGN or in a BCG X-ray coronae. Instead, $K_{0}$ represents the typical excess of core entropy above the best fitting power-law found at larger radii. Cavagnolo et al. (2008a) shows that $K_{0}$ is nonzero for almost all clusters in our sample.

In this letter we present the results of exploring the relationship between the expected by-products of cooling, e.g. $\mathrm{H} \alpha$ emission, star formation, and AGN activity, and the $K_{0}$ values of clusters in our survey. To determine the activity level of feedback in cluster cores, we selected two readily available observables: $\mathrm{H} \alpha$ and radio emission. We have found that there is a critical entropy level below which $\mathrm{H} \alpha$ and radio emission are often present, while above this threshold these emission sources are much fainter and in most cases undetected. Our results suggest that the formation of thermal instabilities in the ICM and initiation of processes such as star formation and AGN activity are closely connected to core entropy, and we suspect that the sharp entropy threshold we have found arises from thermal conduction (Voit et al. 2008, in press).

This letter proceeds in the following manner: In $\$ 2$ we cover the basics of our data analysis. The entropy- $\mathrm{H} \alpha$ relationship is discussed in $\$ 3$, while the entropy-radio relationship is discussed in $\$ 4$. A brief summary is provided in $\$ 5$ For this letter we have assumed a flat $\Lambda$ CDM Universe with cosmogony $\Omega_{\mathrm{M}}=0.3, \Omega_{\Lambda}=0.7$, and $H_{0}=70 \mathrm{~km} \mathrm{~s}^{-1} \mathrm{Mpc}^{-1}$. All uncertainties are $90 \%$ confidence.

\section{DATA ANALYSIS}

This section briefly describes our data reduction and methods for producing entropy profiles. More thorough explanations are given in Donahue et al. (2006), Cavagnolo et al. (2008a), and Cavagnolo et al. (2008b).

$$
\text { 2.1. X-ray }
$$

X-ray data was taken from publicly available observations in the Chandra Data Archive. Following standard CIAO re- 
duction techniques ${ }^{2}$, data was reprocessed using CIAO 3.4.1 and CALDB 3.4.0, resulting in point source and flare clean events files at level-2. Entropy profiles were derived from the radial ICM temperature and electron density profiles.

Radial temperature profiles were created by dividing each cluster into concentric annuli with the requirement of at least three annuli containing a minimum of 2500 counts each. Source spectra were extracted from these annuli, while corresponding background spectra were extracted from blanksky backgrounds tailored to match each observation. Each blank-sky background was corrected to account for variation of the hard-particle background, while spatial variation of the soft-galactic background was accounted for through addition of a fixed background component during spectral fitting. Weighted responses which account for spatial variations of the CCD calibration were also created for each observation. Spectra were then fit over the energy range $0.7-7.0 \mathrm{keV}$ in XSPEC 11.3.2ag (Arnaud 1996) using a single-component absorbed thermal model.

Radial electron density profiles were created using surface brightness profiles and spectroscopic information. Exposurecorrected, background-subtracted, point-source-clean surface brightness profiles were extracted from $5^{\prime \prime}$ concentric annular bins over the energy range $0.7-2.0 \mathrm{keV}$. In conjunction with the spectroscopic normalization and $0.7-2.0 \mathrm{keV}$ count rate, surface brightness was converted to electron density using the deprojection technique of Kriss et al. (1983). Errors were estimated using 5000 Monte Carlo realizations of the surface brightness profile.

A radial entropy profile for each cluster was then produced from the temperature and electron density profiles. The entropy profiles were fit with a simple model which is a powerlaw at large radii and approaches a constant value, $K_{0}$, at small radii (see $\$ 1$ for the equation). We define central entropy as $K_{0}$ from the best-fit model.

\section{2. $\mathrm{H} \alpha$}

One goal of our project was to determine if ICM entropy is connected to processes like star formation. Here we do not directly measure star formation but instead use $\mathrm{H} \alpha$, which is usually a strong indicator of ongoing star formation in galaxies (Kennicutt 1983). It is possible that some of the $\mathrm{H} \alpha$ emission from BCGs is not produced by star formation (Begelman \& Fabian 1990; Sparks et al. 2004; Ruszkowski et al. 2008; Ferland et al. 2008). Nevertheless, $\mathrm{H} \alpha$ emission unambiguously indicates the presence of $\sim 10^{4}$ $\mathrm{K}$ gas in the cluster core and therefore the presence of a multiphase intracluster medium that could potentially form stars.

Our $\mathrm{H} \alpha$ values have been gathered from several sources, most notably Crawford et al. (1999). Additional sources of data are M. Donahue's observations taken at Las Campanas and Palomar (reported in Cavagnolo et al. 2008a), Heckman et al. (1989), Donahue et al. (1992), Lawrence et al. (1996), Valluri \& Anupama (1996), White et al. (1997), Crawford et al. (2005), and Quillen et al. (2008). We have recalculated the $\mathrm{H} \alpha$ luminosities from these sources using our assumed $\Lambda \mathrm{CDM}$ cosmological model. However, the observations were made with a variety of apertures and in many cases may not reflect the full $\mathrm{H} \alpha$ luminosity of the BCG. The exact levels of $L_{\mathrm{H} \alpha}$ are not important for the purposes of this letter and we use the $L_{\mathrm{H} \alpha}$ values here as a binary indicator of multiphase gas: either $\mathrm{H} \alpha$

\footnotetext{
${ }^{2}$ http://cxc.harvard.edu/ciao/guides/
}

emission and cool gas are present or they are not.

\subsection{Radio}

Another goal of this work was to explore the relationship between ICM entropy and AGN activity. It has long been known that BCGs are more likely to host radioloud AGN than other cluster galaxies (Burns et al. 1981; Valentijn \& Bijleveld 1983; Burns 1990). Thus, we chose to interpret radio emission from the BCG of each ACCEPT cluster as a sign of AGN activity.

To make the radio measurements, we have taken advantage of the nearly all-sky flux-limited coverage of the NRAO VLA Sky Survey (NVSS, Condon et al. 1998) and Sydney University Molonglo Sky Survey (SUMSS, Bock et al. 1999; Mauch et al. 2003). NVSS is a continuum survey at 1.4 $\mathrm{GHz}$ of the entire sky north of $\delta=-40^{\circ}$, while SUMSS is a continuum survey at $843 \mathrm{MHz}$ of the entire sky south of $\delta=-30^{\circ}$. The completeness limit of NVSS is $\approx 2.5 \mathrm{mJy}$ and for SUMSS it is $\approx 10 \mathrm{mJy}$ when $\delta>-50^{\circ}$ or $\approx 6 \mathrm{mJy}$ when $\delta \leq-50^{\circ}$. The NVSS positional uncertainty for both right ascension and declination is $\lesssim 1^{\prime \prime}$ for sources brighter than $15 \mathrm{mJy}$, and $\approx 7^{\prime \prime}$ at the survey detection limit (Condon et al. 1998). At $z=0.2$, these uncertainties represent distances on the sky of $\sim 3-20 \mathrm{kpc}$. For SUMSS, the positional uncertainty is $\lesssim 2^{\prime \prime}$ for sources brighter than $20 \mathrm{mJy}$, and is always less than 10" (Bock et al. 1999; Mauch et al. 2003). The distance at $z=0.2$ associated with these uncertainties is $\sim 6-30$ $\mathrm{kpc}$. We calculate the radio power for each radio source using the standard relation $\nu L_{\nu}=4 \pi D_{L}^{2} S_{\nu} f_{0}$ where $S_{\nu}$ is the 1.4 $\mathrm{GHz}$ or $843 \mathrm{MHz}$ flux from NVSS or SUMSS, $D_{L}$ is the luminosity distance, and $f_{0}$ is the central beam frequency of the observations. Our calculated radio powers are simply an approximation of the bolometric radio luminosity.

Radio sources were found using two methods. The first method was to search for sources within a fixed angular distance of $20^{\prime \prime}$ around the cluster X-ray peak. The probability of randomly finding a radio source within an aperture of $20^{\prime \prime}$ is exceedingly low ( $<0.004$ for NVSS). Thus, in 222 total field searches, we expect to find no more than one spurious source. The second method involved searching for sources within 20 projected $\mathrm{kpc}$ of the cluster X-ray peak. At $z \approx 0.051,1^{\prime \prime}$ equals $1 \mathrm{kpc}$, thus for clusters at $z \gtrsim 0.05$, the $20 \mathrm{kpc}$ aperture is smaller than the $20^{\prime \prime}$ aperture, and the likelihood of finding a spurious source gets smaller. Both methods produce nearly identical lists of radio sources with the differences arising from the very large, extended lobes of low-redshift radio sources such as Hydra A.

To make a spatial and morphological assessment of the radio emission's origins, i.e. determining if the radio emission is associated with the BCG, high angular resolution is necessary. However, NVSS and SUMSS are low-resolution surveys with FWHM of $\approx 45^{\prime \prime}$. We therefore cannot distinguish between ghost cavities/relics, extended lobes, point sources, reaccelerated regions, or if the emission is coming from a galaxy very near the BCG. We have handled this complication by visually inspecting each radio source in relation to the optical (using DSS I/II ${ }^{3}$ ) and infrared (using $2 \mathrm{MASS}^{4}$ ) emission of the BCG. We have used this method to establish that the radio emission is most likely coming from the BCG. When available, high resolution data from VLA FIRST ${ }^{5}$ was added to the

\footnotetext{
${ }^{3}$ http://archive.stsci.edu/dss/

4 http://www.ipac.caltech.edu/2mass/

5 http://sundog.stsci.edu
} 


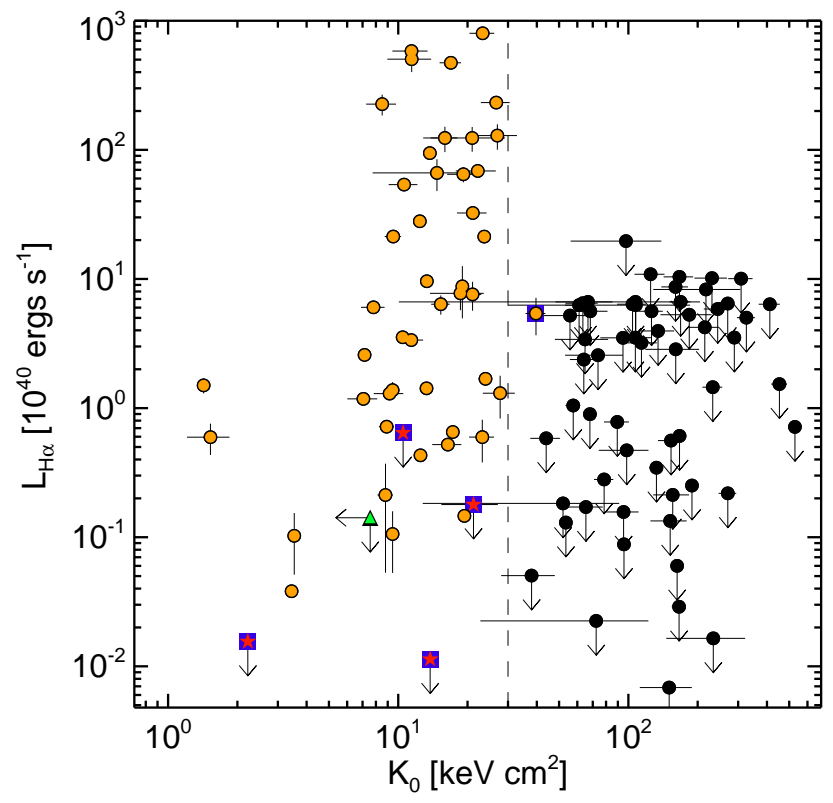

FIG. 1.- Central entropy vs. $\mathrm{H} \alpha$ luminosity. Orange circles represent $\mathrm{H} \alpha$ detections, black circles are non-detection upper limits, and blue squares with inset red stars or orange circles are peculiar clusters which do not adhere to the observed trend (see text). A2151 is plotted using the $2 \sigma$ upper-limit of the best-fit $K_{0}$ and is denoted by a green triangle. The vertical dashed line marks $K_{0}=30 \mathrm{keV} \mathrm{cm}^{2}$. Note the presence of a sharp $\mathrm{H} \alpha$ detection dichotomy beginning at $K_{0} \lesssim 30 \mathrm{keV} \mathrm{cm}{ }^{2}$.

visual inspection. VLA FIRST is a $10,000 \square^{\circ}$ high-resolution $\left(5^{\prime \prime}\right)$ survey at $20 \mathrm{~cm}$ of the North and South Galactic caps (Becker et al. 1995). FIRST is also more sensitive than either NVSS or SUMSS with a detection threshold of $1 \mathrm{mJy}$.

\section{3. $\mathrm{H} \alpha$ EMISSION AND CENTRAL ENTROPY}

Of the 222 clusters in $A C C E P T$, we located $\mathrm{H} \alpha$ observations from the literature for 110 clusters. Of those $110, \mathrm{H} \alpha$ was detected in 46, while the remaining 64 have upper limits. The mean central entropy for clusters with detections is $K_{0}=13.9 \pm 4.9 \mathrm{keV} \mathrm{cm}^{2}$, and for clusters with only upperlimits $K_{0}=130 \pm 55 \mathrm{keV} \mathrm{cm}{ }^{2}$.

In Figure 1 central entropy is plotted versus $\mathrm{H} \alpha$ luminosity. One can immediately see the dichotomy between clusters with and without $\mathrm{H} \alpha$ emission. If a cluster has a central entropy $\lesssim 30 \mathrm{keV} \mathrm{cm}{ }^{2}$ then $\mathrm{H} \alpha$ emission is usually "on", while above this threshold the emission is predominantly "off". For brevity we refer to this threshold as $K_{\text {thresh }}$ hereafter. The cluster above $K_{\text {thresh }}$ which has $\mathrm{H} \alpha$ emission (blue square with inset orange circle) is Zwicky $2701\left(K_{0}=39.7 \pm 3.9 \mathrm{keV} \mathrm{cm}{ }^{2}\right)$. There are also clusters below $K_{\text {thresh }}$ without $\mathrm{H} \alpha$ emission (blue squares with red stars): A2029, A2107, EXO 0422-086, and RBS 533. A2151 also lies below $K_{\text {thresh }}$ and has no detected $\mathrm{H} \alpha$ emission, but the best-fit $K_{0}$ for A2151 is statistically consistent with zero and this cluster is plotted using the $2 \sigma$ upper-limit of $K_{0}$ (green triangle in Fig. 11). These five clusters are clearly exceptions to the much larger trend. The mean and dispersion of the redshifts for clusters with and without $\mathrm{H} \alpha$ are not significantly different, $z=0.124 \pm 0.106$ and $z=0.132 \pm 0.084$ respectively, and applying a redshift cut (i.e. $z=0-0.15$ or $z=0.15-0.3$ ) does not change the $K_{0}$ $\mathrm{H} \alpha$ dichotomy. Most important to note is that changes in the $\mathrm{H} \alpha$ luminosities because of aperture effects will move points up or down in Figure 1, while mobility along the $K_{0}$ axis is minimal. Qualitatively, the correlation between low central

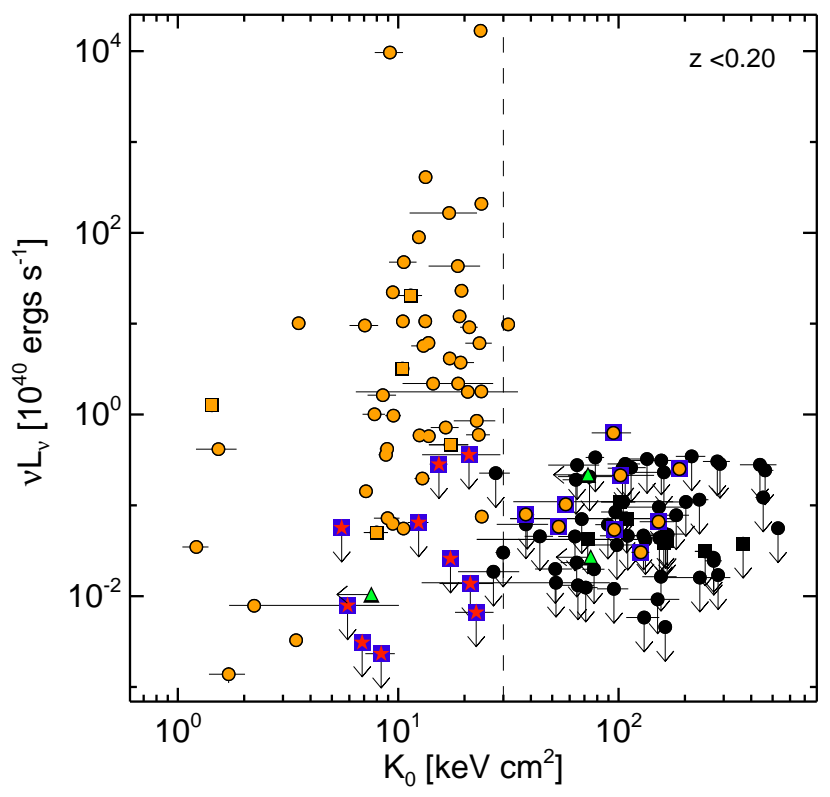

FIG. 2.- BCG radio power vs. $K_{0}$ for clusters with $z<0.2$. Orange symbols represent radio detections and black symbols are non-detection upperlimits. Circles are for NVSS observations and squares are for SUMSS observations. The blue squares with inset red stars or orange circles are peculiar clusters which do not adhere to the observed trend (see text). Green triangles denote clusters plotted using the $2 \sigma$ upper-limit of the best-fit $K_{0}$. The vertical dashed line marks $K_{0}=30 \mathrm{keV} \mathrm{cm}{ }^{2}$. The radio sources show the same trend as $\mathrm{H} \alpha$ : bright radio emission is preferentially "on" for $K_{0} \lesssim 30 \mathrm{keV} \mathrm{cm}{ }^{2}$.

entropy and presence of $\mathrm{H} \alpha$ emission is very robust.

The clusters with $\mathrm{H} \alpha$ detections are typically between $10-30 \mathrm{keV} \mathrm{cm}^{2}$, have short central cooling times ( $<1 \mathrm{Gyr}$ ), and under older nomenclature would be classified as "cooling flow" clusters. It has long been known that star formation and associated $\mathrm{H} \alpha$ nebulosity appear only in cluster cores with cooling times less than a Hubble time ( $\mathrm{Hu}$ et al. 1985; Johnstone et al. 1987; McNamara \& O'Connell 1989; Voit \& Donahue 1997; Cardiel et al. 1998). However, our results suggest that the central cooling time must be at least a factor of 10 smaller than a Hubble time for these manifestations of cooling and star formation to appear. It is also very interesting that the characteristic entropy threshold for strong $\mathrm{H} \alpha$ emission is so sharp. Voit et al. (2008) have recently proposed electron thermal conduction may be responsible for setting this threshold. This hypothesis has received further support from the theoretical work of Guo et al. (2008) showing that thermal conduction can stabilize non-cool core clusters against the formation of thermal instabilities, and that AGN feedback may be required to limit star formation when conduction is insufficient.

\section{RADIO SOURCES AND CENTRAL ENTROPY}

Of the 222 clusters in ACCEPT, 100 have radio-source detections with a mean $K_{0}$ of $23.3 \pm 9.4 \mathrm{keV} \mathrm{cm}^{2}$, while the other 122 clusters with only upper limits have a mean $K_{0}$ of $134 \pm 52 \mathrm{keV} \mathrm{cm}{ }^{2}$. NVSS and SUMSS are low resolution surveys with FWHM at $\approx 45^{\prime \prime}$ which at $z=0.2$ is $\approx 150 \mathrm{kpc}$. This scale is larger than the size of a typical cluster cooling region and makes it difficult to determine absolutely that the radio emission is associated with the $\mathrm{BCG}$. We therefore focus only on clusters at $z<0.2$. After the redshift cut, 135 clusters remain -64 with radio detections (mean $K_{0}=18.3 \pm$ $7.7 \mathrm{keV} \mathrm{cm}^{2}$ ) and 71 without (mean $K_{0}=112 \pm 45 \mathrm{keV} \mathrm{cm}^{2}$ ). 
In Figure 2 we have plotted radio power versus $K_{0}$. The obvious dichotomy seen in the $\mathrm{H} \alpha$ measures and characterized by $K_{\text {thresh }}$, is also present in the radio. Clusters with $\nu L_{\nu} \gtrsim$ $10^{40} \mathrm{erg} \mathrm{s}^{-1}$ generally have $K_{0} \lesssim K_{\text {thresh. }}$. This trend was first evident in Donahue et al. (2005) and suggests that AGN activity in BCGs, while not exclusively limited to clusters with low core entropy, is much more likely to be found in clusters which have a core entropy less than $K_{\text {thresh. That star forma- }}$ tion and AGN activity are subject to the same entropy threshold suggests the mechanism which promotes or initiates one is also involved in the activation of the other. If the entropy of the hot gas in the vicinity of the AGN is correlated with $K_{0}$, then the lack of correlation between radio power and $K_{0}$ below the $30 \mathrm{keV} \mathrm{cm}^{2}$ threshold suggests that cold-mode accretion (Pizzolato \& Soker 2005; Hardcastle et al. 2007) may be the dominant method of fueling AGN in BCGs.

We have again highlighted exceptions to the general trend seen in Figure 2. clusters below $K_{\text {thresh }}$ without a radio source (blue squares with inset red stars) and clusters above $K_{\text {thresh }}$ with a radio source (blue squares with inset orange circles). The peculiar clusters below $K_{\text {thresh }}$ are A133, A539, A1204, A2107, A2556, AWM7, ESO 5520200, MKW4, MS J0440.5+0204, and MS J1157.3+5531. The peculiar clusters above $K_{\text {thresh }}$ are 2PIGG J0011.5-2850, A193, A586, A2063, A2147, A2244, A3558, A4038, and RBS 461. In addition, there are three clusters, A2151, AS405, MS 0116.3-0115, which have best-fit $K_{0}$ statistically consistent with zero and are plotted in Fig. 2] using the $2 \sigma$ upper-limit of $K_{0}$ (green triangles). All three of these clusters have detected radio sources.

Finding a few clusters in our sample without radio sources where we expect to find them is not surprising given that AGN feedback could be episodic. However, the clusters above $K_{\text {thresh }}$ with a central radio source are interesting, and may be special cases of BCGs with embedded coronae. Sun et al.
(2007) extensively studied coronae and found they are like "mini-cooling cores" with low temperatures and high densities. Coronae are a low-entropy environment isolated from the high-entropy ICM and may provide the conditions necessary for gas cooling to proceed. And indeed, 2PIGG 0011, A193, A2151, A2244, A3558, A4038, and RBS 461 show indications that a very compact $(r \lesssim 5 \mathrm{kpc}) \mathrm{X}$-ray source is associated with the BCG (Cavagnolo et al. 2008a).

\section{SUMMARY}

We have presented a comparison of ICM central entropy values and measures of $\mathrm{BCG} \mathrm{H} \alpha$ and radio emission for a Chandra archival sample of galaxy clusters. We find that below a characteristic central entropy threshold of $K_{0} \approx 30 \mathrm{keV} \mathrm{cm}^{2}, \mathrm{H} \alpha$ and bright radio emission are more likely to be detected, while above this threshold $\mathrm{H} \alpha$ is not detected and radio emission, if detected at all, is significantly fainter. The mean $K_{0}$ for clusters with and without $\mathrm{H} \alpha$ detections are $K_{0}=13.9 \pm 4.9 \mathrm{keV} \mathrm{cm}{ }^{2}$ and $K_{0}=130 \pm 55 \mathrm{keV} \mathrm{cm}{ }^{2}$, respectively. For clusters at $z<0.2$ with $\mathrm{BCG}$ radio emission the mean $K_{0}=18.3 \pm 7.7 \mathrm{keV} \mathrm{cm}^{2}$, while for BCGs with only upper limits, the mean $K_{0}=112 \pm 45 \mathrm{keV} \mathrm{cm}^{2}$. While other mechanisms can produce $\mathrm{H} \alpha$ or radio emission besides star formation and $\mathrm{AGN}$, if one assumes the $\mathrm{H} \alpha$ and radio emission are coming from these two feedback sources, then our results suggest the development of multiphase gas in cluster cores (which can fuel both star formation and AGN) is strongly coupled to ICM entropy.

We were supported in this work through $\mathrm{CXO}$ grants AR-6016X, AR-4017A, and NASA LTSA program NNG05GD82G. The CXC is operated by the SAO for and on behalf of NASA under contract NAS8-03060.

\section{REFERENCES}

Arnaud, K. A. 1996, in ASP Conf. Ser. 101: Astronomical Data Analysis Software and Systems V, ed. G. H. Jacoby \& J. Barnes, 17-+

Becker, R. H., White, R. L., \& Helfand, D. J. 1995, ApJ, 450, 559

Begelman, M. C., \& Fabian, A. C. 1990, MNRAS, 244, 26P

Bîrzan, L., Rafferty, D. A., McNamara, B. R., Wise, M. W., \& Nulsen, P. E. J. 2004, ApJ, 607, 800

Bock, D. C.-J., Large, M. I., \& Sadler, E. M. 1999, AJ, 117, 1578

Bower, R. G., Benson, A. J., Malbon, R., Helly, J. C., Frenk, C. S., Baugh, C. M., Cole, S., \& Lacey, C. G. 2006, MNRAS, 370, 645

Burns, J. O. 1990, AJ, 99, 14

Burns, J. O., White, R. A., \& Hough, D. H. 1981, AJ, 86, 1

Cardiel, N., Gorgas, J., \& Aragon-Salamanca, A. 1998, MNRAS, 298, 977

Cavagnolo, K. W., Donahue, M., Sun, M., \& Voit, G. M. 2008a, in prep for ApJ

Cavagnolo, K. W., Donahue, M., Voit, G. M., \& Sun, M. 2008b, ArXiv eprints, arXiv:0803.3858

Condon, J. J., Cotton, W. D., Greisen, E. W., Yin, Q. F., Perley, R. A., Taylor, G. B., \& Broderick, J. J. 1998, AJ, 115, 1693

Crawford, C. S., Allen, S. W., Ebeling, H., Edge, A. C., \& Fabian, A. C. 1999, MNRAS, 306, 857

Crawford, C. S., Hatch, N. A., Fabian, A. C., \& Sanders, J. S. 2005, MNRAS, 363,216

Croton, D. J., Springel, V., White, S. D. M., De Lucia, G., Frenk, C. S., Gao, L., Jenkins, A., Kauffmann, G., Navarro, J. F., \& Yoshida, N. 2006, MNRAS, 365, 11

Donahue, M., Horner, D. J., Cavagnolo, K. W., \& Voit, G. M. 2006, ApJ, 643 , 730

Donahue, M., Stocke, J. T., \& Gioia, I. M. 1992, ApJ, 385, 49

Donahue, M., Voit, G. M., O’Dea, C. P., Baum, S. A., \& Sparks, W. B. 2005 , ApJ, 630, L13

Ferland, G. J., Fabian, A. C., Hatch, N. A., Johnstone, R. M., Porter, R. L., van Hoof, P. A. M., \& Williams, R. J. R. 2008, MNRAS, 386, L72
Guo, F., Oh, S. P., \& Ruszkowski, M. 2008, ArXiv e-prints, arXiv:0804.3823 Hardcastle, M. J., Evans, D. A., \& Croston, J. H. 2007, MNRAS, 376, 1849 Heckman, T. M., Baum, S. A., van Breugel, W. J. M., \& McCarthy, P. 1989, ApJ, 338, 48

Hu, E. M., Cowie, L. L., \& Wang, Z. 1985, ApJS, 59, 447

Johnstone, R. M., Fabian, A. C., \& Nulsen, P. E. J. 1987, MNRAS, 224, 75 Kennicutt, Jr., R. C. 1983, ApJ, 272, 54

Kriss, G. A., Cioffi, D. F., \& Canizares, C. R. 1983, ApJ, 272, 439

Lawrence, C. R., Zucker, J. R., Readhead, A. C. S., Unwin, S. C., Pearson, T. J., \& Xu, W. 1996, ApJS, 107, 541

Mauch, T., Murphy, T., Buttery, H. J., Curran, J., Hunstead, R. W., Piestrzynski, B., Robertson, J. G., \& Sadler, E. M. 2003, MNRAS, 342, 1117

McNamara, B. R., \& Nulsen, P. E. J. 2007, ARA\&A, 45, 117

McNamara, B. R., \& O’Connell, R. W. 1989, AJ, 98, 2018

Peterson, J. R., \& Fabian, A. C. 2006, Phys. Rep., 427, 1

Pizzolato, F., \& Soker, N. 2005, ApJ, 632, 821

Quillen, A. C., Zufelt, N., Park, J., O’Dea, C. P., Baum, S. A., Privon, G., Noel-Storr, J., Edge, A., Russell, H., Fabian, A., Donahue, M., Bregman, J. N., McNamara, B. R., \& Sarazin, C. L. 2008, ApJS, 176, 39

Ruszkowski, M., Enßlin, T. A., Brüggen, M., Begelman, M. C., \& Churazov, E. 2008, MNRAS, 383, 1359

Saro, A., Borgani, S., Tornatore, L., Dolag, K., Murante, G., Biviano, A., Calura, F., \& Charlot, S. 2006, MNRAS, 373, 397

Sparks, W. B., Donahue, M., Jordán, A., Ferrarese, L., \& Côté, P. 2004, ApJ, 607, 294

Sun, M., Jones, C., Forman, W., Vikhlinin, A., Donahue, M., \& Voit, M. 2007, ApJ, 657, 197

Valentijn, E. A., \& Bijleveld, W. 1983, A\&A, 125, 223

Valluri, M., \& Anupama, G. C. 1996, AJ, 112, 1390

Voit, G. M. 2005, Reviews of Modern Physics, 77, 207 
Voit, G. M., Bryan, G. L., Balogh, M. L., \& Bower, R. G. 2002, ApJ, 576, 601

Voit, G. M., Cavagnolo, K. W., Donahue, M., Rafferty, D. A., McNamara, B. R., \& Nulsen, P. E. J. 2008, ArXiv e-prints, arXiv:0806.0384
Voit, G. M., \& Donahue, M. 1997, ApJ, 486, 242

White, D. A., Jones, C., \& Forman, W. 1997, MNRAS, 292, 419 